\title{
Centered Pyramids
}

\author{
Patrick Brigger, Member, IEEE, Frank Müller, Student Member, IEEE, \\ Klaus Illgner, Member, IEEE, and Michael Unser, Fellow, IEEE
}

\begin{abstract}
Quadtree-like pyramids have the advantage of resulting in a multiresolution representation where each pyramid node has four unambiguous parents. Such a centered topology guarantees a clearly defined up-projection of labels. This concept has been successfully and extensively used in applications of contour detection, object recognition and segmentation. Unfortunately, the quadtree-like type of pyramid has poor approximation powers because of the employed piecewise-constant image model.

This paper deals with the construction of improved centered image pyramids in terms of general approximation functions. The advantages of the centered topology such a symmetry, consistent boundary conditions and accurate up-projection of labels are combined with a more faithful image representation at coarser pyramid levels. We start by introducing a general framework for the design of least squares pyramids using the standard filtering and decimation tools. We give the most general explicit formulas for the computation of the filter coefficients by any (well behaving) approximation function in both the continuous $\left(L_{2}\right)$ and the discrete $\left(l_{2}\right)$ norm. We then define centered pyramids and provide the filter coefficients for odd spline approximation functions. Finally, we compare the centered pyramid to the ordinary one and highlight some applications.
\end{abstract}

Index Terms-Haar pyramid, multiresolution decomposition, multiscale processing, pyramids.

\section{INTRODUCTION}

$\mathbf{M}$ ULTISCALE processing is an old but powerful idea [7], [16]. It is usually applicable whenever one wishes to implement an image processing algorithm that is iterative in nature and requires many successive updates. The basic principle is to construct an image pyramid and to start applying the procedure at the coarsest level on a very small version of the image. Upon convergence, the solution is propagated to the next finer level where it is used as starting condition. One then proceeds with this coarse-to-fine iteration strategy until one reaches the finest level of the pyramid which corresponds to the image itself. This type of multiresolution approach has

Manuscript received March 5, 1997; revised September 10, 1998. This project was supported in part by the Swiss National Research Foundation The associate editor coordinating the review of this manuscript and approving it for publication was Dr. Dapang Chen.

P. Brigger was with the Biomedical Engineering and Instrumentation Program, National Center for Research Resources, National Institutes of Health, Bethesda, MD 20892-5766 USA. He is now with ImageMinds, Greenwich, CT 06830 USA (e-mail: pbrigger@imageminds.com).

F. Müller is with Aachen University of Technology, Aachen D-52056 Aachen, Germany.

$\mathrm{K}$. Illgner is with the DSPS R\&D Center, Texas Instruments, Dallas, TX 75265 USA.

M. Unser is with the Biomedical Imaging Group, Swiss Federal Institute of Technology, CH-1015 Lausanne, Switzerland.

Publisher Item Identifier S 1057-7149(99)06830-X. two advantages. First, it decreases the number of computations since most iterations are spent processing reduced versions of the image. Second, it usually also improves robustness; the pyramid has a smoothing effect on the criterion to be optimized which often reduces the likelihood of getting trapped in local optima.

There are numerous examples of the application of this principle in the literature. Bouman et al. [5] employ a quadtreelike pyramid for unsupervised texture segmentation and Kato et al. [12] uses a multiscale relaxation algorithm applied to image classification. Pyramids have also been used in [4] to compute time-varying motion parameters and in [18], a Haar pyramid is used for segmentation of moving objects. A Haar pyramid of processors is used in [11] for efficient exploration of the Hough parameter space for line detection. Thévenaz et al. [20] employ a multiresolution strategy for the computation of displacement parameters.

Recently, there has been a renewed interest in pyramids because of the key role of these structures in the multiresolution theory of the wavelet transform [8], [13], [19], [27]. One of the main concerns there is data compression and the multiresolution decomposition is usually optimized for maximum energy compaction [21], [22], [26]. While the wavelet theory has resulted in the construction of improved image pyramids, most multiscale algorithms that assign image labels still operate with the oldest and most primitive form: the nearest neighbor or Haar pyramid. The main reason for this is that this pyramid has a nice centered (quadtree-like) topology in which each coarser level node is situated exactly in the center of its finer level predecessors. This is in contrast with most other pyramids (Gaussian or spline-based) which use symmetric reduction and expansion filters so that there is an ambiguity when one attempts to propagate an image label (not a pixel) from one level to the next (Fig. 1). Naturally, a one-pixel wide label should lead to a two-pixel wide label at the finer level that has twice the resolution of the coarser level. The centered topology guarantees a clearly defined way of propagating labels across scales. This advantage has been exploited in [5], [11], [12], [18] which employ quadtree-like pyramids. The down side of the Haar pyramid, however, is that it has very poor approximation power because the underlying model is piecewise constant, hence one operates on a bad replica of the original image at coarser resolutions.

The purpose of this paper is to correct for this deficiency of the Haar pyramid by introducing higher order extensions that preserve the centered topology and provide more accurate representations of the original image at coarser resolution levels. 


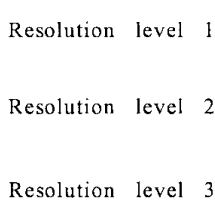

Resolution level 3

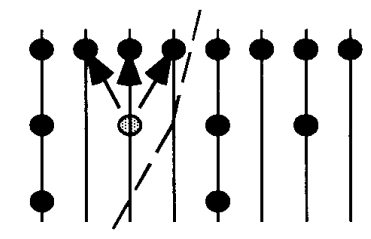

(a)

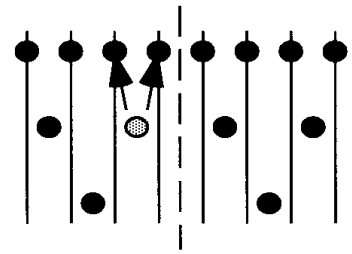

(b)

Fig. 1. (a) Ambiguous situation for up-projection of a label in the ordinary pyramid. (b) Symmetric up-projection with the centered pyramid.

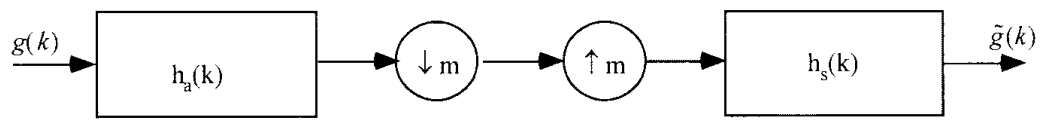

Fig. 2. Original signal is reduced by filtering and downsampling, the extrapolated signal is obtained by upsampling and filtering.

In addition, the centered topology assures that the image center at resolution (i) maps onto the image center at resolution $(i+$ $1)$, thus simplifying multiresolution motion or displacement parameter estimation because of a common spatial reference system. Also, the centered pyramid offers an accurate way of up-projection because it is symmetric. Applications include multiscale contour detection, object recognition/detection and segmentation, and potentially also zerotree pyramid coding. Furthermore, with the introduction of appropriate boundary conditions, the resulting signal has a periodicity of $2 N$ rather than $2 N-2$. This means that 1) fast Fourier transforms (FFT's) are easier to use and 2) the boundary conditions are mutually compatible at all levels.

The construction of a pyramid requires the repeated application of a reduction function that reduces the (rectangular) image sizes by a factor of $m$ and the image content by a factor of $m^{2}$. The reverse operation is obtained via an expansion function which extrapolates a coarse image representation onto a finer one. This procedure is outlined in Fig. 2. The reduce operation is typically implemented by filtering and downsampling. Similarly, the expand function is implemented by upsampling and filtering (i.e., interpolation). The filter $h_{a}(k)$ is called the analysis filter whereas the filter $h_{s}(k)$ is called the synthesis filter. The analysis filter should be designed in such a way as to minimize the loss of information occurring during resolution conversion, and the synthesis filter should be designed in such a way as to minimize the approximation error between the original image and the extrapolated one [25]. Moreover, in the type of pyramid we are introducing here, the grid of the coarser representation is shifted with respect to the finer one so that the pyramid is centered.

The paper is organized as follows. After some brief definitions in Section II, we derive the general pyramid formulas in both the continuous and the discrete framework for arbitrary generating functions (Section III). Note that in contrast with the conventional wavelet theory, our multiresolution spaces are not necessarily nested. In Section IV, we define the concept of centered pyramid and derive explicit transfer functions using polynomial splines. We discuss the problem of boundary
TABLE I

Basic Discrete Signal Processing Operators AND THEIR EFFECT IN THE $z$-DomaIN

\begin{tabular}{ccc}
\hline Operator & Notation & Z-transform \\
\hline Inner product: & $\langle a(k), b(k)\rangle_{\ell_{2}}=\sum_{-\infty}^{+\infty} a(k) b(k)=a * b^{r}(0)$ & \\
Convolution & $(a * b)(k)=\sum_{i \in Z} a(l) b(k-l)$ & $A(z) \cdot B(z)$ \\
Time-shift & $a\left(k-k_{0}\right)=\sum_{l \in Z}\left(\delta_{k_{n}}^{*} a\right)(k)$ & $A(z) \cdot z^{-k_{0}} \quad k_{0} \in Z$ \\
Time-reversal & $a^{\gamma}(k)=a(-k)$ & $A(1 / z)$ \\
Up-sampling & {$[a]_{\uparrow m}(k)$} & $A\left(z^{m}\right)$ \\
Down-sampling & {$[a]_{\downarrow_{m}}(k)$} & $\frac{1}{m} \sum_{k \cdot 0}^{m-1} A\left(\left[z e^{j 2, k}\right]^{1 / m}\right)$ \\
Convolution inverse & $(a)^{-1}(k)$ & $1 / A(z)$ \\
\hline
\end{tabular}

extension in Section $\mathrm{V}$ and highlight some applications in Section VI.

\section{DEFINITIONS}

Our developments are a generalization of the work performed by Unser et al. in [25] for the continuous case and of the work by Aldroubi et al. in [1] for the discrete case. We adopt the same notations as in these references. Hence, $L_{2}$ denotes the space of measurable, square integrable functions $g(x), x \in R ; l_{2}$ is the vector space of square summable sequences $a(k), k \in Z$. We will use a variety of operators that act on discrete $l_{2}$ sequences; these are summarized in Table I. Note that the whole theory is developed in one dimension. The extension to multidimensional signals such as images is straightforward. We simply use separable (tensor product) basis functions and implement the algorithm (reduction or expansion) by successive one-dimensional (1-D) processing of the various dimensions along the data.

\section{Generalized Least SQuares Pyramids}

Our objective is to derive the synthesis and analysis transfer functions for a centered pyramid construction. In this section, 
however, formulas by more general basis functions will be developed (for any shift of the coarser grid), since it does not complicate our task and only clarifies the mathematics. Appropriate basis functions yielding a centered pyramid will be given in the next section. First, the continuous case will be considered and then the discrete case, which is completely analogous.

Let us consider a step-wise optimal approximation from a fine space $S_{1}$ to a coarse space $S_{2}$. These spaces are generated by dilated an shifted basis functions, such that

$$
S_{1}\left(\varphi_{1}(x)\right)=\left\{g_{1}(x)=\sum_{k \in Z} c_{1}(k) \varphi_{1}(x-k), c_{1} \in l_{2}\right\}
$$

$S_{2}\left(\varphi_{2}\left(\frac{x}{m}\right)\right)=\left\{g_{2}(x)=\sum_{k \in Z} c_{2}(k) \varphi_{2}\left(\frac{x}{m}-k\right), c_{2} \in l_{2}\right\}$.

The approximation of the original signal becomes coarser as we employ larger dilations and larger shifts of the basis functions. A multiresolution pyramid may be constructed in an iterative way by considering coarser spaces of basis functions dilated and shifted by multiples of $m$. Typically, $m=2$, but there is no theoretical difficulty in considering other integer reduction factors. We propose to compute the expansion coefficients $c_{2}(k)$ in (2) by digital filtering. Digital filtering means that we require the expansion coefficients $c_{2}(k)$ of a coarse representation level to be specified by the expansion coefficients $c_{1}(k)$ of the finer resolution level. Furthermore, the generating functions $\varphi_{1}$ and $\varphi_{2}$ must satisfy

$$
0<A \leq \sum_{k \in Z}\left|\hat{\varphi}_{i}(\omega+2 \pi k)\right|^{2} \leq B<\infty
$$

where $\hat{\varphi}_{i}(\omega)$ denotes the Fourier transform of $\varphi_{i}(x)$, i.e., $\varphi_{1}$ and $\varphi_{2}$ must generate a Riesz basis. Condition (3) guarantees that the spaces $S_{i}$ are closed in $L_{2}$ and that a least squares approximation of a function $g(x) \in L_{2}$ into $S_{i}$ exists [2]. The conditions for multiresolution decomposition in $L_{2}$ have been defined in [13]. In the theory of wavelet transforms, the spaces are self-similar and their union over all scales is dense in $L_{2}$. Our conditions here are weaker; no nestedness between approximation spaces is required and $S_{1}$ and $S_{2}$ may even use different basis functions. The originality of our approach is that it will be possible to use more general basis functions that can be tailored to specific requirements. In Section IV, we will use shifted spline basis functions to obtain a centered pyramid that has several nice properties. Next, we derive the pyramid transfer functions in the general case.

\section{A. Analysis Function (REDUCE Operation)}

We now turn to the issue of finding the best approximation of a function $g_{1}(x) \in S_{1}$ into the coarser subspace $S_{2}$ by minimizing a least squares criterion. The minimal $L_{2^{-}}$ norm approximation of a signal $g_{1}(x) \in S_{1}$ is obtained by orthogonally projecting $g(x)$ into $S_{2}$. Thus, the expansion coefficients $c_{2}(k)$ in (2) must be obtained from the ones given in (1).
Let $\varphi_{1}(x)$ be a generating function in $L_{2}$ that spans $S_{1}$ and let $\varphi_{2}(x / m)$ be another generating function that spans $S_{2}$, dilated by a factor $m$ and completely independent from $\varphi_{1}(x)$. Before stating our first result, we define the following correlation coefficients:

$$
\begin{aligned}
& a_{i j}(k)=\left\langle\varphi_{i}(x), \varphi_{j}(x-k)\right\rangle \\
& q_{i j}(k)=\left\langle\varphi_{i}(x), \varphi_{j}\left(\frac{x-k}{m}\right)\right\rangle \\
& r_{i j}(k)=\left\langle\varphi_{i}\left(\frac{x}{m}\right), \varphi_{j}(x-k)\right\rangle .
\end{aligned}
$$

The following theorem provides a solution to the general $L_{2}$ approximation problem:

Theorem 1: Let $\left\{\varphi_{1}(x-k), k \in Z\right\}$ and $\left\{\varphi_{2}(x / m-\right.$ $k), k \in Z\}$ be two sets of Riesz basis functions of $S_{1}$ and $S_{2}$, respectively. The orthogonal projection of the function $g_{1}(x) \in S_{1}$

$$
g_{1}(x)=\sum_{k \in Z} c_{1}(k) \varphi_{1}(x-k)
$$

into $S_{2}$ is given by

$$
g_{2}(x)=\sum_{k \in Z} c_{2}(k) \varphi_{2}\left(\frac{x}{m}-k\right)
$$

where the expansion coefficients $c_{2}(k)$ are obtained as:

$$
c_{2}(k)=\frac{1}{m}\left[\left[a_{22}(k)\right]_{\uparrow m}^{-1} * q_{12} * c_{1}\right]_{\downarrow m}(k) .
$$

The proof is given in Appendix A. In the $z$-transform domain, the transfer function is written as

$$
H_{A}(z)=\frac{1}{m} \frac{Q_{12}(z)}{A_{22}\left(z^{m}\right)}
$$

where $Q_{12}(z)$ and $A_{22}(z)$ are the $z$-transform of $q_{12}(k)$ and $a_{22}(k)$, respectively.

Sometimes it is convenient to compute (9) and (10) for some basis functions, but to represent the signal $g(x)$ with other basis functions (e.g., cardinal spline representation). This can be done with the coordinate transformation expressed in the following corollary.

Corollary 1: Let

$$
\begin{aligned}
& g_{1}(x)=\sum_{k \in Z} \tilde{c}_{1}(k) \tilde{\varphi}_{1}(x-k) \quad \text { and } \\
& g_{2}(x)=\sum_{k \in Z} \tilde{c}_{2}(k) \tilde{\varphi}_{2}(x / m-k)
\end{aligned}
$$

where

$$
\tilde{\varphi}_{i}(k)=\sum_{k \in Z} p_{i}(k) \varphi_{i}(x-k) .
$$

Then

$$
\begin{aligned}
\tilde{c}_{2}(k) & =\frac{1}{m}\left[\left[a_{22} * p_{2}(k)\right]_{\uparrow m}^{-1} * q_{12} * c_{1} * p_{1}\right]_{\downarrow m}(k) . \\
\tilde{H}_{A}(z) & =\frac{1}{m} \frac{Q_{12}(z)}{A_{22}\left(z^{m}\right)} \frac{P_{1}(z)}{P_{2}\left(z^{m}\right)} .
\end{aligned}
$$


Given the special case where a link from the coarse scale to the fine scale exists ( $m$-scale relation)

$$
\varphi_{2}\left(\frac{x}{m}\right)=\sum_{k \in Z} u_{m}(k) \varphi_{0}(x-k)
$$

then (12) and (13) may be further simplified by replacing the cross-correlation term $q_{12}(k)$ by $\left(u_{m}\right)^{T} * a_{10}(k)$. Here, we have introduced an auxiliary function $\varphi_{0}(x)$ which may be distinct from $\varphi_{1}(x)$, (e.g., it may be a shifted version by half a sampling step). Note that (14) is more general than the two-scale relation encountered in the theory of the wavelet transform: $\varphi_{0}$ is not necessarily identical to $\varphi_{2}$ and $m$ is not necessarily a power of two.

\section{B. Synthesis Function (EXPAND Operation) in $L_{2}$}

To go in the other direction, i.e., expanding a resolution level, the approximation in the finer scale is achieved by orthogonal projection of the coarser space into the finer space. Note that this process, which guarantees a minimum loss of information, is equivalent to an interpolation when the spaces are nested.

Theorem 2: Given the same settings as in Theorem 1, the orthogonal projection of a function $g_{2}(x) \in S_{2}$

$$
g_{2}(x)=\sum_{k \in Z} c_{2}(k) \varphi_{2}\left(\frac{x}{m}-k\right)
$$

into $S_{1}$ is given by

$$
g_{1}(x)=\sum_{k \in Z} c_{1}(k) \varphi_{1}(x-k)
$$

where the expansion coefficients $c_{1}(k)$ are obtained as:

$$
c_{1}(k)=\left[a_{11}(k)\right]^{-1} * r_{21}(k) *\left[c_{2}\right]_{\uparrow m}(k) .
$$

The proof is given in Appendix B. The corresponding synthesis transfer function is therefore

$$
H_{S}(z)=\frac{R_{21}(z)}{A_{11}(z)} .
$$

In the same way as for the reduction operation, the transfer function can be represented using the $\mathrm{m}$-scale relation and a transformed coordinate system:

$$
\begin{aligned}
\tilde{c}_{1}(k) & =\left[a_{11} * p_{1}(k)\right]^{-1} * u_{m} * a_{01} *\left[c_{2} * p_{2}\right]_{\uparrow m}(k) \\
H_{S}(z) & =\frac{U_{m}(z) \cdot A_{01}(z)}{A_{11}(z)} \frac{P_{2}\left(z^{m}\right)}{P_{1}(z)}
\end{aligned}
$$

where $p_{i}$ 's are the sequences in (11).

\section{Analysis and Synthesis Function in the Discrete Space $\left(l_{2}\right)$}

The formal derivations of the discrete transfer functions are completely analogous to the continuous case. We now consider discrete Riesz basis in a space

$$
V_{m}=\left\{g(k)=\sum_{l \in Z} c(l) \cdot h_{m}(k-m l)\right\}
$$

where the link with the continuous formulation is $h_{m}(k)=$ $\left.\varphi_{2}(x)\right|_{x=k / m}$.
Theorem 3: Let $g(k) \in l_{2}$ be a discrete function and $\left\{h_{m}(k-m l), l \in Z\right\}$ a Riesz basis of a discrete space $V_{m}$. The orthogonal projection of $g(k)$ into $V_{m}$ is

$$
g_{m}(k)=\sum_{j \in Z} c_{2}(j) h_{m}(k-m j)
$$

where the coefficients $c_{2}(k)$ are given by

$$
c_{2}(k)=\left[\left[h_{m} *\left(h_{m}\right)^{T}\right]_{\downarrow m \uparrow m} * g *\left(h_{m}\right)^{T}\right]_{\downarrow m}(k)
$$

Proof: The prove follows the one given in Appendix A. The $l_{2}$-orthogonality of the error implies that

$$
\begin{aligned}
\left\langle g(l), h_{m}(l-m k)\right\rangle_{l_{2}} & =\left\langle\sum_{j \in Z} c_{2} h_{m}(l-m j), h_{m}(l-m k)\right\rangle_{l_{2}} \\
\forall k \in Z & \\
{\left[g *\left(h_{m}\right)^{T}\right]_{\downarrow m}(k) } & =c_{2} *\left[h_{m} *\left(h_{m}\right)^{T}\right]_{\downarrow m}(k)
\end{aligned}
$$

which gives (22). Notice that we have used the fact that $\left\lfloor a_{\uparrow m} * b\right\rfloor_{\downarrow m}=a *[b\rfloor_{\downarrow m}$. The developments are analogous to the ones for (9), except that we can not further simplify the correlation product $h_{m} *\left(h_{m}\right)^{T}(k)$ (in the continuous case, it leads to autocorrelation).

In the $z$-domain, the transfer function of the equivalent analysis filter is

$$
H_{A}(z)=\stackrel{\circ}{H}_{m}(z)=\frac{H_{m}(1 / z)}{1 / m \sum_{k=0}^{m-1} H_{m}\left(W^{k} z\right) \cdot H_{m}\left(W^{k} z^{-1}\right)}
$$

with $W^{k}=e^{j 2 \pi k / m}$. By definition, the corresponding resulting approximation is given by $g(k)=\left[c_{2}\right]_{\uparrow m} * h_{m}(k)$. Thus the transfer function of the expansion filter is simply $H_{s}(z)=H_{m}(z)$.

\section{Centered Pyramid}

In this section, we define the centered pyramid and give examples using polynomial splines.

\section{A. Definition of Centered Pyramid}

We will only consider basis functions with an axis of symmetry. These correspond to the nodes of the pyramid. In this way, there is no phase distortion and the objects in the image are not shifted. Both conventions are crucial if we want to correctly maintain the localization of objects in the coarser levels of the pyramid. From now on, we will only consider pyramids with a scale reduction of two $(m=2)$.

Definition 1: For a centered pyramid, the basis functions must satisfy

(i) $\varphi_{1}(x)=\varphi_{1}(-x)$

(ii) $\varphi_{2}\left(\frac{x+1}{2}\right)=\varphi_{2}\left(\frac{-x}{2}\right)$

(iii) $\varphi_{0}(x)=\varphi_{1}\left(x-\frac{1}{2}\right)$ 
where $\varphi_{2}(x / 2)=\Sigma_{k \in Z} u(k) \cdot \varphi_{0}(x-k)$. Note that (ii) and (iii) imply that $u(k)=u(-k)$.

A centered pyramid exhibits clear advantages in a number of typical image analysis problems that involve the up-projection of labels. These advantages stem mainly from the propriety of symmetry (Fig. 1). As an example, we may consider the important problem of contour detection or segmentation, where a one pixel wide boundary needs to be extrapolated to a finer scale. In a scale reduction of two, naturally, the extrapolated boundary must be two pixels wide. It is immediately given by a centered pyramid, whereas an ambiguous situation subsides in the noncentered case.

Since the greatest part of the energy in an image is contained in the DC component, we also impose a perfect reconstruction of the constant. In the continuous framework, this is equivalent to requiring that

$$
\sum_{k \in Z} \varphi_{2}(x-2 k)=1 \quad \text { (partition of the unity). }
$$

In the discrete case, the condition is

$$
\sum_{l \in Z} h_{2}(k-2 l)=1 \text {. }
$$

It is well known in wavelet theory that (29) is equivalent to $H\left(e^{j \pi}\right)=0$ [19]. We will show here that the same condition is also necessary and sufficient in the discrete case. The left side of (30) is periodic with period two, and therefore can be represented by two samples of its continuous Fourier transformation. Therefore, $\Sigma_{l \in Z} h_{2}(k-2 l)=H\left(e^{j 0}\right)+$ $H\left(e^{j \pi}\right) \cdot \cos (\pi k)=1, \forall k \in Z$, which is true if and only if $H\left(e^{j \pi}\right)=0$ and $H\left(e^{j 0}\right)=1$.

Proposition 1: For a centered pyramid

$$
\begin{aligned}
& H_{S}(z)=z^{-1} H_{S}\left(z^{-1}\right) . \\
& H_{A}(z)=z \cdot H_{A}\left(z^{-1}\right) .
\end{aligned}
$$

Proof: Let us consider the synthesis transfer function first. For the discrete case, $h_{m}(k)=\left.\varphi_{2}(x / m)\right|_{x=k}$ and (31) follows directly from (27). In the continuous case, $H_{S}(z)$ is given by (18). The autocorrelation function is symmetric, and thus we only need to characterize $r_{21}(k)$.

$$
\begin{aligned}
r_{21} & =\int_{-\infty}^{+\infty} \varphi_{2}(x / 2) \cdot \varphi_{1}(x-k) d x \\
& =\int_{-\infty}^{+\infty}\left[\sum_{j \in Z} u(j) \cdot \varphi_{0}(x-j)\right] \cdot \varphi_{1}(x-k) d x \\
& =\int_{-\infty}^{+\infty}\left[\sum_{j \in Z} u(j) \cdot \varphi_{1}(x-1 / 2-j)\right] \cdot \varphi_{1}(x-k) d x \\
& =\sum_{j \in Z} u(j) \cdot \int_{-\infty}^{+\infty} \varphi_{1}(x-1 / 2-j) \cdot \varphi_{1}(x-k) d x \\
& =\left.\sum_{j \in Z} u(j) \cdot\left(\varphi_{1} * \varphi_{1}^{T}\right)(x-1 / 2-j)\right|_{x=k} .
\end{aligned}
$$

This signal is symmetric with respect to a shift by half a pixel. This implies that $r_{21}(k+1)=r_{21}(-k)$ and proves (31). The developments for the analysis function are analogous. In the discrete case, we may also use (31) as a definition.
Proposition 2: Given a centered pyramid for which $H(z)$ is a stable rational transfer function (ratio of two polynomials) and which reproduces the constant, then

$H(z)=\left(1+z^{-1}\right) \cdot H_{\mathrm{sym}}(z) \quad$ with $\quad H_{\mathrm{sym}}(z)=H_{\mathrm{sym}}\left(z^{-1}\right)$

where $H_{\mathrm{sym}}(z)$ is a stable symmetric transfer function.

Proof: If $H(z)$ is rational, then $H\left(e^{j \pi}\right)=0$ implies that

$$
H(z)=\left(1+z^{-1}\right) \cdot Q(z)
$$

has a zero at $z=-1$ and $Q(z)$ is stable (i.e., no root at $z=-1)$. The fact that $Q(z)$ is symmetric then follows from Proposition 1.

Next, we will present explicit examples of centered pyramids using polynomial splines.

\section{B. Definition of Polynomial Splines}

Spline polynomial functions [17] have been shown to be approximation functions with nice properties such as regularity, least oscillation, smoothness [15]. The symmetrical B-spline of order zero is defined as:

$$
\beta^{0}(x)= \begin{cases}1, & \text { for }|x| \leq 1 / 2 \\ 0, & \text { for }|x|>1 / 2\end{cases}
$$

The continuous B-spline function of degree $n$ is denoted by $\beta^{0}(x)$. It can be constructed using the important convolution property

$$
\beta^{n}(x)=\underbrace{\beta^{0} * \beta^{0} * \cdots * \beta^{0}(x)}_{n+1 \text { times }} .
$$

The discrete version and shifted discrete version of splines is obtained by sampling the continuous one

$$
\begin{aligned}
b^{n}(k / m) & =\left.\beta^{n}(x / m)\right|_{x=k} \\
d^{n}(k / m) & =\left.\beta^{n}((x+1 / 2) / m)\right|_{x=k} .
\end{aligned}
$$

\section{Transfer Functions for Centered Pyramids}

Constructing the filter coefficients for a spline pyramid means replacing $\varphi_{1}(x)$ and $\varphi_{2}(x)$ in (12) by spline functions. On all levels of the pyramid, splines are imposed and furthermore, we want the grid of the coarser level to be centered; i.e., shifted by one-half with respect to the grid of the finer level. Hence, the changes become

$$
\begin{aligned}
\varphi_{1}(x) & =\beta^{n}(x) \\
\varphi_{2}\left(\frac{x}{2}\right) & =\beta^{n}\left(\frac{x-1 / 2}{2}\right) .
\end{aligned}
$$

For an easy visualization of the discrete image, we will choose the coefficients $c_{1}(k)$ and $c_{2}(k)$ to be the discrete data points of the signal $g(k)=\left.g(x)\right|_{x=k}$ at each resolution level. This representation is known as the cardinal spline representation. It determines the function $p_{1}(k)$ and $p_{2}(k)$ in (11) which are given in [25], as follows:

$$
p_{1}(k)=p_{2}(k)=\left[b^{n}\right]^{-1}(k) .
$$


TABLE II

Analysis and Synthesis Transfer Functions in the Transformed Coordinate System for $n$ OdD

\begin{tabular}{|c|c|c|}
\hline Filter type & Impulse response & Frequency response \\
\hline Continuous: & $\frac{1}{2}\left[\left(b^{2 n+1}\right)^{-1} * b^{n}(k)\right]_{\uparrow 2} * u_{2}^{n} * d^{2 n+1}(k) *\left(b^{n}\right)^{-1}(k)$ & $\left(\frac{1+z}{2}\right) \frac{U_{2}^{n}(z) \cdot E^{2 n+1}(z)}{B^{2 n+1}\left(z^{2}\right)} \frac{B^{n}\left(z^{2}\right)}{B^{n}(z)}$ \\
\hline Synthesis & {$\left[b^{2 n+1} *\left[b^{n}\right]_{\uparrow 2}\right]^{-1} * b^{n} * u_{2}^{n} * d^{2 n+1}(k-1)$} & $\left(1+z^{-1}\right) \frac{U_{2}^{n}(z) \cdot E^{2 n+1}(z)}{B^{2 n+1}(z)} \frac{B^{n}(z)}{B^{n}\left(z^{2}\right)}$ \\
\hline Discrete: Analysis & {$\left[u_{2}^{n} * d^{2 n+1} * u_{2}^{n} *\left(d^{2 n+1}\right)^{\gamma}\right]_{\downarrow_{2} \uparrow 2}^{-1} * u_{2}^{n} *\left(d^{n}\right)^{T} *\left[b^{n}\right\rfloor_{t_{2}}(k)$} & 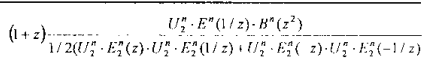 \\
\hline Synthesis & $u_{2}^{n} * d^{n} *\left[b^{n}\right]_{\uparrow 2}^{-1}(k)$ & $\left(1+z^{-1}\right) \frac{U_{2}^{n} \cdot E^{n}(z)}{B^{n}\left(z^{2}\right)}$ \\
\hline
\end{tabular}

Furthermore, a two-scale relation exists for spline functions [23], which is given for $n$ being odd as

$U_{m}^{n}(z)=\frac{z^{(n+1) / 2}}{2^{n}}\left(1+z^{-1}\right)^{(n+1)} \quad$ and $\quad \varphi_{0}(x)=\beta^{n}(x)$.

We obtain for the various convolution products ( $n$ odd):

$$
\begin{aligned}
a_{22}(k)= & \int_{-\infty}^{+\infty} \beta^{n}(x-1 / 2) \beta^{n}(x-1 / 2-k) d x \\
= & b^{2 n+1}(k) \\
q_{12}(k)= & \int_{-\infty}^{+\infty} \beta^{n}(x) \\
& \cdot\left[\sum_{j \in Z} u_{2}^{n}(j) \beta^{n}(x-1 / 2-j-k)\right] d x \\
= & \left(u_{2}^{n}\right)^{T} * d^{2 n+1}(k) \\
r_{21}(k)= & \int_{-\infty}^{+\infty}\left[\sum_{j \in Z} u_{2}^{n}(j) \beta^{n}(x-1 / 2-j)\right] \\
& \cdot \beta^{n}(x-k) ; d x \\
= & u_{2}^{n} * d^{2 n+1}(k-1) .
\end{aligned}
$$

The transfer functions for the discrete and continuous case can now be formulated and they are given in Table II in the time domain and in the $z$-domain (convention: $d^{n}(k) \leftrightarrow^{z}(1+$ $z) \cdot E^{n}(z)$ and $b^{n}(k) \leftrightarrow^{z} B^{n}(z)$, and, for $n$ odd, $\left.\left(u_{2}^{n}\right)^{T}=u_{2}^{n}\right)$.

The transfer functions can be computed for $n=$ even in an analogous way. Then, $\varphi_{0}(x)=\beta^{n}(x+1 / 2)$ and $U_{2}^{n}(x)=\left(z^{n / 2} / 2^{n}\right)\left(1+z^{-1}\right)^{n+1}$.

The corresponding pyramid filters are infinite impulse response (IIR), and can be expressed as sums of exponentials. Such a direct computation of the filter coefficients is not practical. Instead, it is much simpler to pass through the Fourier domain. Our strategy will be as follows.

1) Express the transfer functions in the $z$-domain.

2) Replace $z$ by $e^{-j 2 \pi f}$ to switch to the Fourier domain.
3) Sample the Fourier domain with a sampling step high enough to avoid significant time-domain aliasing.

4) Compute the inverse discrete Fourier transform to obtain the filter coefficients.

These computations can be easily carried out with software packages such as Mathematica or Matlab. Implementation is done using truncated filters with an effective length that is even (odd length convolved with Haar). In Fig. 3(a) and (b), a plot of the transfer functions in the frequency domain for the continuous case is shown, for $m=2$, and $n=1,2,3$. The analysis filter acts as antialiasing filter and the synthesis filter plays the role of the interpolation filter. An exact computation of the impulse response may be obtained using a recursive computation.

The transfer functions for centered pyramids as defined above are for spline functions of any odd order of $n$. A spline of order zero corresponds to the Haar pyramid; it seems intuitively obvious that a centered pyramid of higher order will perform better than the Haar pyramid. In Table III, those expectations are experimentally verified. For a number of different images, the ordinary and centered $l_{2}$ pyramid was computed performing one reduction operation followed by an expansion operation. Then, the peak signal-to-noise ratio (PSNR) is computed comparing the up-projected image to the original one. The centered pyramid compares well to the noncentered one, performing always better or at least as good. As we approach higher spline orders, $n>=3$, the differences in PSNR become insignificant. As a matter of fact, the centered and noncentered pyramids have identical performance for an infinite order cardinal spline, which corresponds to the sinc interpolator and which is shift-invariant [3]. However, the centered pyramid is preferable because of the properties mentioned before. Since we are dealing exclusively with digital pictures, we have considered the discrete image pyramid. Splines also compare favorably with respect to Gaussian pyramids [24], which have not been considered here anymore. The centered topology is also optimal with respect to a shiftinvariance measure, which has been introduced and studied elsewhere [14]. 


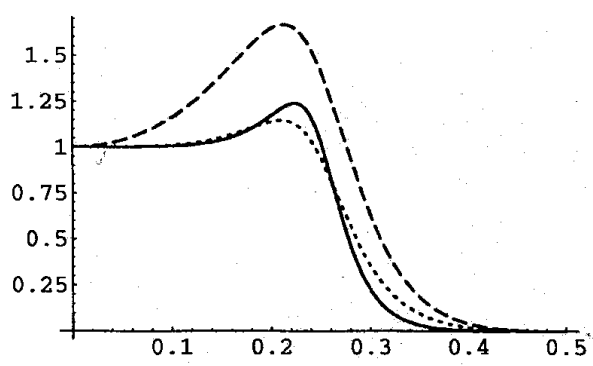

(a)

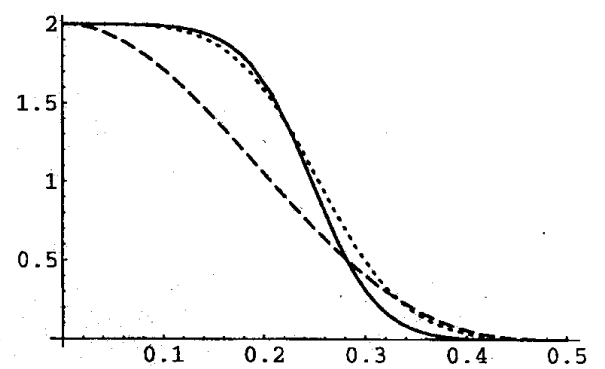

(b)

Fig. 3. Transfer functions for spline pyramid. (Dashed: degree 1. Dotted: degree 2. Solid: degree 3). (a) Analysis function. (b) Synthesis function.

TABLE III

Comparison of PSNR BetweEn the HaAR, Ordinary and CEnTEREd Pyramid

\begin{tabular}{ll|r|r|r|r}
\hline & & \multicolumn{3}{|c}{ Peak signal to noise ratio (PSNR) } \\
& & \multicolumn{3}{|c}{ [dB] } \\
\hline Pyramid type & & MRI & Lena & MIT & Camera \\
& & brain & & building & man \\
& & image & & & \\
\hline \hline Haar & & 27.8 & 26.5 & 26.5 & 24.1 \\
\hline Ordinary (non-centered) & order 1 & 32.6 & 28.1 & 28.4 & 24.1 \\
\hline \multirow{2}{*}{ Centered } & order 3 & 35.2 & 28.6 & 29.0 & 25.8 \\
\hline & order 1 & 34.1 & 28.5 & 28.7 & 25.6 \\
\hline & order 3 & 35.2 & 28.6 & 29.0 & 25.7 \\
\hline
\end{tabular}

\section{BORDER CONDITIONS}

In the above developments, the signals were generally assumed to be of infinite length. Such a hypothesis is not valid anymore for real signals and we need to define appropriate boundary extensions to extend the finite signal to infinite length. The boundary conditions have to fulfill two requirements:

1) the boundaries at resolution level $i$ must be computed from the signal values at level $i$;

2) the reduced (expanded) boundaries from level $i(i-1)$ must be the same as the boundaries computed at the next lower (higher) level $(i-1) i$.

In other words, the boundaries should not be affected by the discrete convolution operator of the filter. Traditional boundary extensions such as mirroring or antimirroring do not fulfill these requirements. A valid boundary extension is obtained by mirroring with respect to a symmetry axis at the end of the interval shifted by half a pixel off the limit signal values, as depicted in Fig. 4 (half-sample symmetry [6]).

In the case of a centered pyramid, it was shown that the analysis and synthesis filter can be decomposed into a symmetric component $H_{\text {sym }}(z)$ and a Haar component $\left(1+z^{-1}\right)$. The validity of the proposed boundary extension can then be proven as follows. It is easily verified that the Haar filter by itself fulfills the boundary extensions requirements. Indeed, the

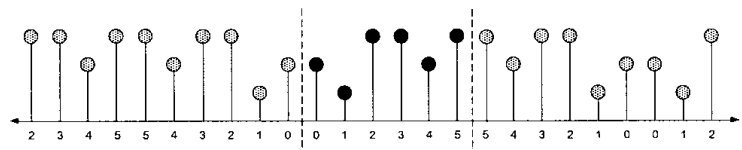

Fig. 4. Black dots represent the original signal; gray dots represent the extended boundaries. The mirror axis is between the grid points.

averaging operations on either side of the symmetry axis are identical. As far as the remaining component is concerned, it suffices to realize that the symmetric filter component produces the same result for equidistant pixels on each side of the symmetry axis, as is obvious from Fig. 4 . Hence, the boundary conditions are valid for any centered approximation. They can be formalized as follows. We denote by $g(k), k \leq 0<N$ the original signal of length $N$ and by $g_{s}(k)$ the extended signal of infinite length.

$$
\begin{aligned}
g_{s}(k) & = \begin{cases}g\left(k_{s}\right), & \text { if } k_{s}<N \\
f\left(-k_{s}-1+2 N\right), & \text { otherwise. }\end{cases} \\
k_{s} & = \begin{cases}k \bmod 2 N, & \text { if } k \geq 0 \\
(2 N-1-k) \bmod 2 N, & \text { if } k<0 .\end{cases}
\end{aligned}
$$

Note that these boundary conditions are of periodicity $2 N$, which may be advantageous for FFT based implementations. Furthermore, a periodicity $2 N$ assures consistent boundary conditions across scales, which is not the case in ordinary pyramids with symmetric boundary extensions, for instance.

The filter coefficients (discrete or continuous) may be downloaded via FTP. Also, a C-program is available that implements the border extension mechanism and computes the centered pyramid in the 1-D and two-dimensional (2-D) case. The pyramid decomposition for images is obtained by applying the filters in the direction of the rows first, and then by applying the filters in the directions of the columns. The C-routines can be accessed in the public directory of the anonymous ftp site: picasso.ncrr.nih.gov.

\section{Results AND APPLICATION}

Because of their centering properties, these new pyramids should provide an interesting alternative for all multiscale applications that use quadtree-like representations or Haar pyramids. The centered pyramids should provide better or at least identical results for any application involving pyramids. 

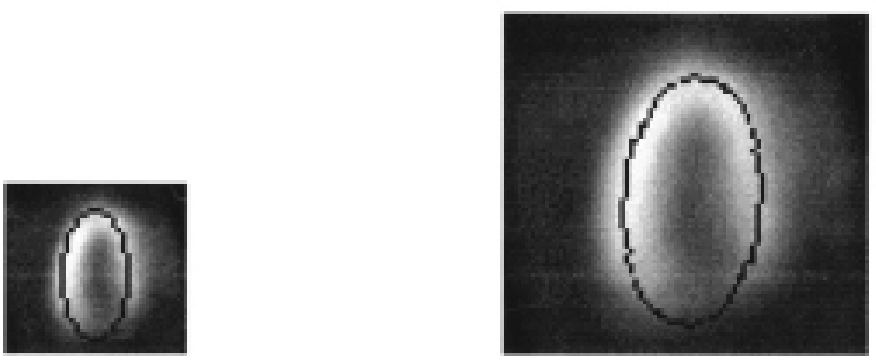

Fig. 5. Multiresolution Hough parameter fitting.

In particular, they should be useful for applications wherever a result at a coarser resolution level needs to be projected upwards onto a finer resolution level where the processing continues. Such applications include edge detection, motion estimation, and parameter fitting.

\section{A. Multiresolution Hough Parameter Fitting}

The Hough algorithm is used to fit curves to binary signals [9]. Every variable in the analytical curve model defines one dimension in the multidimensional Hough space. The Hough space is then scanned for all possible parameter values. For each bin in the Hough space, the number of signal points that are covered by the model are counted. The bin with the maximal counts represents the parameters of the unknown curve model.

This algorithm is very simple, but very time consuming. Computation time is proportional to the square of the image size and is proportional to the number of curve parameters to be estimated. Since we can not affect the second factor, we can substantially decrease the amount of processing time by a multiresolution approach. With this implementation, the bin with maximal counts in the Hough space of a spatially reduced signal is computed. This result then needs to be symmetrically up-projected onto the next finer level, of which the result is immediately given by the centered pyramid. At this level, the optimal result is situated in the neighborhood of the upprojected pixels, and it is therefore sufficient to only compute the number of counts in the neighboring bins.

An example is given in Fig. 5. The image shows a SPECT scan (single-photon emission computed tomography) of a long axis slice of the heart, where the myocardium is imaged. The ultimate imaging goal is to find the endo- and epi-cardial borders. Because of the ellipse like coordinates of the heart, image processing is facilitated if the heart is expressed in ellipsoidal coordinates rather than in Cartesian coordinates. We have employed the $l_{2}$ centered cubic spline pyramid and the Hough algorithm to find the optimal short and long axes and the center of the ellipse. The fitted ellipse is superimposed in black on each of the resolutions in the image of Fig. 5. The search for the elliptical parameters involves the computation of five parameters (center, radii and orientation). A pyramid decomposition into three levels enormously speeds up the computation and still allows us to find the correct ellipse. Experimentally, we have determined three pyramid levels to be optimal, as it optimizes speed and still guarantees an
TABLE IV

Comparison OF COMPUTATION TIME

\begin{tabular}{c||c|c|c|c}
\hline \multicolumn{1}{c||}{} & \multicolumn{4}{c}{ Computation time [sec] } \\
\hline \hline Resolution levels & Level 1 & Level 2 & Level 3 & Total \\
\hline 1 & 10400 & & & 10400 \\
\hline 2 & 32 & 65 & & 98 \\
\hline 3 & 32 & 20 & 1 & 53 \\
\hline
\end{tabular}

accurate enough approximation of the original image at the coarsest pyramid level. Computation time for different choices of number of pyramid levels are reported in Table IV. The image size is $128 \times 128$ pixels, and computation were done on a low end workstation (120 MHz PowerPC). The algorithm has been tested on more than 100 images, and in all of them, the correct ellipse was found.

\section{B. Multiresolution Image Registration}

The general problem of image registration concerns the matching of two images minimizing an a priori chosen disparity criterion. The problem arises for example when two images have to be compared and one of them has been translated with respect to the other. Typically, no closed-form expression for the motion parameters exists and the global disparity minimum has to be found iteratively. In a bottom-up multiresolution approach, two pyramids are computed for the original image and the test image, respectively [20]. The algorithm starts at the coarsest resolution and finds the best fit. The result is upprojected onto the next finer level until the original image is reached.

In the case of the affine transformation, one has to fix a center of origin of the coordinate system at each resolution level. The up-projection from one level to the next involves an adjustment of the affine parameters by a scale factor. In addition, if the origins at different levels are not identical, an additional translational term is required, which complicates the computation. The centered pyramid offers a straightforward way of up-projection, reducing some computational complexity and reducing considerably the risk of errors in the program code.

\section{Motion Compensation}

For subpel motion compensation, the current image as well as the reference image are interpolated [10]. On these expanded frames, motion estimation and compensation is per- 
TABLE V

Motion COMPENSATED PSNR OBTAINED for Test Image Using DifFerent Filters

\begin{tabular}{l|lc}
\hline \hline Interpolation function & \multicolumn{2}{|c}{ PSNR [db] } \\
& Test image & Video sequence \\
& 20.92 & foreman \\
\hline Bilinear & 23.12 & 34.8733 \\
Spline, $n=1$ & 24.23 & 36.0868 \\
Spline, $n=1$, centered & 24.76 & 36.3422 \\
Spline, $n=3$ & 24.78 & 36.4567 \\
Spline, $n=3$, centered & &
\end{tabular}

formed and the predicted frame is reduced subsequently to the original size. Typically, bilinear interpolation is used followed by simple subsampling. In fact, subsampling can already be included in the match criterion avoiding the interpolation of the current frame. The use of spline filters provides an improvement both in terms of the prediction gain and in a more accurate motion vector field. The best results in terms of prediction gain has been achieved by using the centered spline filters. For simulations a test image pair has been generated, consisting of a noise texture. To simulate half pel displacements, the texture has been displaced by an odd number of pels and subsequently filtered and subsampled with a standard lowpass filter.

For expansion and reduction the separable $l_{2}$ spline filters are employed. The four filter configurations analyzed are: firstand third-order and both filter centered as well as noncentered. Motion estimation and compensation was performed on the expanded frames using a standard block-oriented approach with full pel accuracy (Table V).

Obviously, the prediction gain obtained using spline filters is significant. Moreover, for the first-order pyramid centering increases the prediction approximately by $1 \mathrm{~dB}$. For centered third-order splines the number of inaccurate motion vectors was lowest. The highest motion compensation gain was achieved by the centered cubic splines.

In case of natural image pairs (frames from a video scene), the accuracy of the motion vector field can not be judged, since the true displacements are unknown. However, the motion compensation gain can be observed. In Table V, the mean motion compensation gain is given, obtained by averaging over 17 frame pairs of the test sequence foreman in QCIF format.

For both filter orders, an improvement of the prediction gain for the centered filters over the noncentered filters can be observed. As before, centering is especially beneficial for lower order splines. The best results are obtained with the centered cubic spline which provides a $0.7-1 \mathrm{~dB}$ improvement over the noncentered first order pyramid. Note that this latter is already $0.8 \mathrm{~dB}$ better than the traditional method that uses bilinear interpolation.

\section{CONCLUSIONS}

In this paper, we have presented the concept of centered pyramids that fulfill the three requirements of 1) minimization of loss of information during resolution conversion, 2) minimization of approximation error during extrapolation, and 3 ) perfect centering of resolution levels. We have provided general formulas for the analysis and synthesis filters in both the continuous and discrete spaces. The only requirement imposed is that the generating basis functions must form Riesz-bases. The obtained formulas may then be used by any specific basis functions. The cubic B-spline basis functions were employed as a specific example for the computation of the filter coefficients. The explicit transfer functions in the $z$-domain are given. We have justified the need for centered pyramids because of their symmetry and periodicity of $2 N$, which result in better properties for image analysis. Also, in the type of pyramid proposed, the center at low resolution levels get perfectly mapped onto finer resolution levels, and the centered pyramid is left/right and up/down flip invariant. We have presented applications that benefit from the multiresolution analysis by centered pyramids, because of speed consideration, better accuracy and the ease of up-projection of parameters. This pyramid can be used as a good substitute in any multiscale algorithm that performs the reduction by averaging (Haar pyramid).

\section{APPENDIX A}

Let us define the correlation products as in (4)-(6). Given two spaces $S_{1}\left(\varphi_{1}(x)\right)$ and $S_{2}\left(\varphi_{2}(x / m)\right)$, the orthogonal projection of $g_{1}(x) \in S_{1}\left(\varphi_{1}\right)$ onto $S_{2}\left(\varphi_{2}\right)$ such that the approximation error is orthogonal to $S_{2}\left(\varphi_{2}\right)$ is defined through the equation

$$
\begin{aligned}
& \left\langle\sum_{l \in Z} c_{1}(l) \varphi_{1}(x-l)-\sum_{j \in Z} c_{2}(j) \varphi_{2}(x / m-j),\right. \\
& \left.\varphi_{2}(x / m-k)\right\rangle_{L_{2}}=0 \quad \forall k \in Z .
\end{aligned}
$$

It is solved as follows:

$$
\begin{aligned}
& \left\langle\sum_{l \in Z} c_{1}(l) \varphi_{1}(x-l), \varphi_{2}(x / m-k)\right\rangle_{L_{2}} \\
& =\left\langle\sum_{j \in Z} c_{2}(j) \varphi_{2}(x / m-j), \varphi_{2}(x / m-k)\right\rangle_{L_{2}} \\
& \forall k \in Z \\
& \sum_{l \in Z} c_{1}(l)\left\langle\varphi_{1}(x-l), \varphi_{2}((x-k) / m)\right\rangle_{\downarrow m} \\
& =\sum_{j \in Z} c_{2}(j)\left\langle\varphi_{2}(x / m-j), \varphi_{2}((x-k) / m)\right\rangle_{\downarrow m} \\
& {\left[\sum_{l \in Z} c_{1}(l) \cdot q_{12}(k-l)\right]_{\downarrow m}} \\
& =m \cdot \sum_{j \in Z} c_{2}(j) \cdot\left[a_{22}(k / m-j)\right]_{\downarrow m} \\
& {\left[c_{1} * q_{12}\right]_{\downarrow m}(k)=m \cdot c_{2} * a_{22}(k) .}
\end{aligned}
$$


The step from the third to the fourth line is obtained by realizing that

$$
\begin{aligned}
& \int \varphi_{2}(x / m-j) \cdot \varphi_{2}((x-k) / m) d x \\
& \stackrel{u=x / m}{=} m \int_{-\infty}^{+\infty} \varphi_{2}(u) \varphi_{2}(u-k / m) d u
\end{aligned}
$$

The downsampling can then be omitted by substituting $k$ for $k / m$. Hence, we obtain (9), q.e.d.

\section{APPENDIX B}

The development for the expansion function is similar to the one for the reduction function given in Appendix A. Given two spaces $S_{1}\left(\varphi_{1}(x)\right)$ and $S_{2}\left(\varphi_{2}(x / m)\right)$, the orthogonal projection of $g_{2}(x) \in S_{2}\left(\varphi_{2}\right)$ onto $S_{1}\left(\varphi_{1}\right)$ must satisfy the orthogonality requirement

$$
\begin{aligned}
& \left\langle\sum_{l \in Z} c_{1}(l) \varphi_{1}(x-l)-\sum_{j \in Z} c_{2}(j) \varphi_{2}(x / m-j),\right. \\
& \left.\varphi_{1}(x-k)\right\rangle_{L_{2}}=0 \quad \forall k \in Z .
\end{aligned}
$$

Solving for $c_{1}(k)$ we get

$$
\begin{aligned}
& \sum_{l \in Z} c_{1}(l)\left\langle\varphi_{1}(x-l), \varphi_{1}(x-k)\right\rangle \\
& =\sum_{j \in Z} c_{2}(j)\left\langle\varphi_{2}((x-m j) / m), \varphi_{1}(x-k)\right\rangle \\
& \forall k \in Z \\
& \sum_{l \in Z} c_{1}(l) \cdot a_{11}(k-l)=\sum_{j \in Z} c_{2}(j) \cdot r_{21}(k-m j) \quad \forall k \in Z \\
& c_{1} * a_{11}(k)=\left[c_{2}\right]_{\uparrow m} * r_{21}(k)
\end{aligned}
$$

and we get the result of (17), q.e.d.

\section{ACKNOWLEDGMENT}

The authors are very grateful to A. Aldroubi for his most valuable suggestions and help on filter design, and to $\mathrm{P}$. Thévenaz for his help in B-spline implementation.

\section{REFERENCES}

[1] A. Aldroubi, M. Eden, and M. Unser, "Discrete spline filters for multiresolution and wavelets of $l_{2}$," SIAM J. Math. Anal., vol. 25, pp. 1412-1432, Sept. 1994.

[2] A. Aldroubi and M. Unser, "Sampling procedures in function spaces and asymptotic equivalence with shannon's sampling theory," Numer. Funct. Anal. Optimiz., vol. 15, pp. 1-21,, 1994.

[3] A. Aldroubi, M. Unser, and M. Eden, "Cardinal spline filters: Stability and convergence to the ideal sinc interpolator," Signal Process., vol. 28 , pp. 127-138, 1992.

[4] S. Ayer and P. Schroeter, "Hierarchical robust motion estimation for segmentation of moving objects," in Proc. 8th IEEE Workshop on Image and Multidimensional Signal Processing, Cannes, France, Sept. 1993.
[5] C. Bouman and B. Liu, "Multiple resolution segmentation of textured images," IEEE Trans. Pattern Anal. Machine Intell., vol. 13, pp. 99-113, Feb. 1991.

[6] C. M. Brislawn, "Preservation of subband symmetry in multirate signal coding," IEEE Trans. Signal Processing, vol. 43, no. 12, pp. 3046-3050, Dec. 1995.

[7] P. J. Burt and E. H. Adelson, "The Laplacian pyramid as a compact code," IEEE Trans. Commun., vol. COMM-31, pp. 337-345, Apr. 1983.

[8] I. Daubechies, Ten Lectures on Wavelets. Philadelphia: SIAM, 1992.

[9] R. O. Duda and P. E. Hart, "Use of the Hough transformation to detect lines and curves," Commun. ACM, vol. 15, pp. 11-15, 1972.

[10] B. Girod, "Motion-compensating prediction with fractional-Pel accuracy," IEEE Trans. Commun., vol. 41, pp. 604-611, 1993.

[11] J. M. Jolion and A. Rosenfeld, "An $O(\log n)$ pyramid Hough transform," Pattern Recognit. Lett., vol. 9, pp. 343-349, June 1989.

[12] Z. Kato, M. Berthold, and J. Zerubia, "A hierarchical markov random field model and multitemperature annealing for parallel image classification," Graph. Models Image Process., vol. 58, pp. 18-37, Jan. 1996.

[13] S. G. Mallat, "A theory of multiresolution signal decomposition: the wavelet representation," IEEE Trans. Pattern Anal. Machine Intell., vol. PAMI-11, pp. 674-693, 1989.

[14] F. Mueller, P. Brigger, K. Illgner, and M. Unser, "Multiresolution approximation using shifted splines," IEEE Trans. Signal Processing, 1996, vol. 46, pp. 2555-2558, Sept. 1998.

[15] P. M. Prenter, Splines and Variational Methods. New York: Wiley, 1975.

[16] A. Rosenfeld, Multiresolution Image Processing. Berlin, Germany: Springer-Verlag, 1984.

[17] I. J. Schoenberg, "Cardinal interpolation and spline functions," $J$ Approx. Theory, vol. 2, pp. 167-206, 1969.

[18] P. Schroeter and J. Bigun, "Hierarchical image segmentation by multidimensional clustering and orientation-adaptive boundary refinement," Pattern Recognit., vol. 28, pp. 695-709, 1995.

[19] G. Strang and T. Nguyen, Wavelets and Filter Banks. WellesleyCambridge Press, 1996.

[20] P. Thévenaz, U. E. Ruttimann, and M. Unser, "Iterative multi-scale registration without landmarks," in Proc. IEEE Int. Conf. Image Processing, Washington, DC, Oct. 23-26, 1995, vol. III, pp. 228-231.

[21] M. Unser, "An improved least squares Laplacian pyramid for image compression," Signal Process., vol. 27, pp. 187-203, May 1992.

[22] _ "On the optimality of ideal filters for pyramid and wavelet signal approximation,” IEEE Trans. Signal Processing, vol. 41, pp. 3591-3596, Dec. 1993.

[23] M. Unser, A. Aldroubi, and M. Eden, "B-spline signal processing. Part I: Theory," IEEE Trans. Signal Processing, vol. 41, pp. 821-833, Feb. 1993.

[24] _ , "B-spline signal processing. Part II: Efficient design and applications," IEEE Trans. Signal Processing, vol. 41, pp. 834-848, Feb. 1993.

[25] $\_$,The $L_{2}$ polynomial spline pyramid," IEEE Trans. Pattern Anal. Machine Intell., vol. 15, pp. 364-379, 1993.

[26] K. M. Uz, M. Vetterli, and D. J. LeGall, "Interpolative multiresolution coding of advanced television with compatible subchannel," IEEE Trans. Circuits Syst. Video Technol., vol. 1, pp. 86-99, Mar. 1991.

[27] M. Vetterli and J. Kovacevic, Wavelets and Subband Coding. Englewood Cliffs, NJ: Prentice-Hall, 1995.

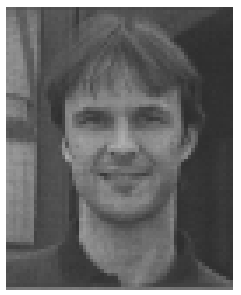

Patrick Brigger (M'96) was born in Lucerne, Switzerland, on May 5, 1966. He received the M.S. (awarded the Jean Laundry Prize) and $\mathrm{Ph} . \mathrm{D}$. degrees in electrical engineering in 1992 and 1995, respectively, both from the Swiss Federal Institute of Technology, Lausanne, Switzerland.

From 1996 to 1998 , he was with the Biomedical Engineering and Instrumentation Program, National Institutes of Health, Bethesda, MD. He directed several projects related to biomedical image processing. In 1998, he became Principal Investigator for a Small Business Innovation Research grant, and became partner of ImageMinds, an imaging and internet technology company. His main field of activity is biomedical engineering, internet technologies, and object-oriented program design. 


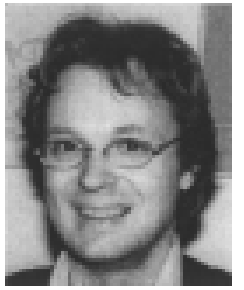

Frank Müller (S'92) received the Diploma degree (M.S.) in electrical engineering from Aachen University of Technology (RWTH), Aachen, Germany, in 1991, and the Ph.D. degree (with distinction) from the Institute of Communications Engineering, RWTH, in 1999. His doctorate was on pyramids for image compression.

Since then, he has been with the Institute of Communications Engineering, RWTH, as a Scientific Assistant His current interests are source coding theory as well as hierarchical and multiresolution approaches to image and video compression.

Dr. Müller is a member of the IEEE Signal Processing Society, the IEEE Information Theory Society, the Association of German Electrical Engineers, and the German Computer Society (Gesellschaft für Informatik).

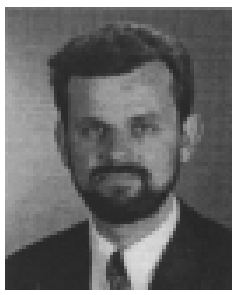

Klaus Illgner (M'98) received the Diploma and $\mathrm{Ph} . \mathrm{D}$. degrees, both in electrical engineering, from Aachen University of Technology (RWTH), Aachen, Germany, in 1991 and 1998, respectively. From 1991 to 1998, he was with the Institute for Communication Engineering, RWTH, as a Scientific Assistant, responsible for an industrial project in the area of video compression and video communications. His research interests focused later on motion compensation and multiresolution video. Since 1998, he has been with the DSPS R\&D Center, Texas Instruments, Dallas, TX. His research interests concentrate on algorithm development for and design of programmable imaging systems.

Dr. Illgner is a member of the German Electrical Engineering Association.

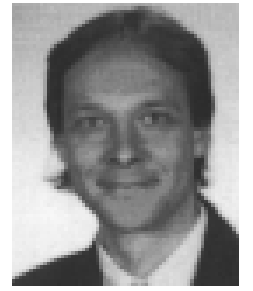

Michael Usner (M'89-SM'94-F'99) was born in Zug, Switzerland, on April 9, 1958. He received the M.S. (summa cum laude) and the Ph.D. degrees in electrical engineering in 1981 and 1984 respectively, from the Swiss Federal Institute of Technology, Lausanne, Switzerland.

From 1985 to 1997 , he was with the Biomedical Engineering and Instrumentation Program, National Institutes of Health, Bethesda, MD, where he was head of the Image Processing Group. He is now Professor and Head of the Biomedical Imaging Group, Swiss Federal Institute of Technology, Lausanne, Switzerland. His main research area is biomedical image processing. He has a strong interest in sampling theories, multiresolution algorithms, wavelets, and the use of splines for image processing. He is the author of over 70 published journal papers in these areas.

Dr. Usner is on the editorial boards of Signal Processing, the Journal of Visual Communication and Image Representation, and Pattern Recognition. He is a former Associate Editor for the IEEE TRANSACTIONS ON IMAGE PROCESSING (1992-1995) and the IEEE Signal Processing LetTERs (1994-1998). He coorganized the 1994 IEEE-EMBS Workshop on Wavelets in Medicine and Biology, and serves as conference chair for SPIE's Wavelet Applications in Signal and Image Processing, which has been held annually since 1993 . He is a member of the Image and Multidimensional Signal Processing Committee of the IEEE Signal Processing Society. He received the Dommer Prize for Excellence from the Swiss Federal Institute of Technology in 1981, the Research Prize of the Brown-Boveri Corporation (Switzerland) for his thesis in 1984, and the IEEE Signal Processing Society's 1995 Best Paper Award (IMDSP technical area) for a Transactions paper (with A. Aldroubi and M Eden) on B-spline signal processing. 